\title{
The Strange Quark Mass From Finite Energy Sum Rules
}

\author{
Kim Maltman \\ Department of Mathematics and Statistics, York University, \\ 4700 Keele St., Toronto, Ontario, CANADA M3J 1P3 \\ and \\ Special Research Centre for the Subatomic Structure of Matter, \\ University of Adelaide, Australia 5005
}

\begin{abstract}
The strange quark mass is determined from a study of the correlator $\left\langle 0\left|T\left(J_{s} J_{s}^{\dagger}\right)\right| 0\right\rangle$ (where $J_{s} \equiv \partial_{\mu} \bar{s} \gamma^{\mu} u$, the divergence of the strange vector current) using a family of finite energy sum rules recently shown to be very accurately satisfied in the isovector vector channel. It is shown that the match between the OPE and hadronic representations for the sum rules employed is extremely good once one fixes the overall scale, $\left(m_{s}-m_{u}\right)^{2}$, which normalizes the OPE side. A value $m_{s}\left(4 \mathrm{GeV}^{2}\right)=115 \pm 8 \mathrm{MeV}$, corresponding to $m_{s}\left(1 \mathrm{GeV}^{2}\right)=159 \pm 11 \mathrm{MeV}$, is obtained, with theoretical and experimental uncertainties contributing roughly equally to the combined error.
\end{abstract}

12.15.Ff,11.55.Hx

Typeset using REVTEX 


\section{INTRODUCTION}

The light quark masses, despite being fundamental parameters of the Standard Model, are surprisingly poorly known at present. Since, in the standard ("large condensate") scenario for the chiral counting in Chiral Perturbation Theory (ChPT), however, the ratio $m_{s} /\left(m_{u}+m_{d}\right)=24.4 \pm 1.5$ is known [1], fixing either $m_{s}$ or $m_{u}+m_{d}$ is sufficient to determine the other. In this paper we focus on $m_{s}$, which has been the subject of several recent investigations, employing both QCD sum rules [2 [11] and lattice QCD [12 223.

On the lattice, reliable results are presently restricted to the quenched approximation and vary somewhat depending on the fermion implementation and on whether one chooses $m_{K}$ or $m_{\phi}$ when matching to physical meson masses. Unquenched calculations are at a more preliminary stage, but suggest unquenching is likely to lower quenched results [12, 16, 17, 20]. Recent quenched results for $m_{s}$ (where here, as in what follows, all masses, unless otherwise specified, will be quoted at a scale $\mu=2 \mathrm{GeV}$ in the $\overline{M S}$ scheme) are $110 \pm 20 \pm 11 \mathrm{MeV}$ [12], $122 \pm 20 \mathrm{MeV}$ [13], $95 \pm 16 \mathrm{MeV}$ [15], $115 \pm 2(143 \pm 6) \mathrm{MeV}$ [17] (for $m_{K}\left(m_{\phi}\right)$ input), $111 \pm 12 \mathrm{MeV}$ [18], $98.1 \pm 2.4 \mathrm{MeV}$ [19] (update as quoted by Kenway [20]), $130 \pm 2 \pm 18 \mathrm{MeV}$ [21], $129 \pm 12 \mathrm{MeV}$ [22] and $95 \pm 26 \mathrm{MeV}$ [23]. For dynamical $\left(N_{f}=2\right)$ simulations, the CPPACS results $m_{s} \sim 70(80) \mathrm{MeV}$ (with $m_{K}\left(m_{\phi}\right)$ as input) and SESAM result $151 \pm 30 \mathrm{MeV}$ [16] do not appear compatible. (Note, however, that there are unresolved issues of chiral extrapolations, renormalization constants, and the lack of extrapolation to the continuum limit in the SESAM result; for a further discussion of these issues see Section 6 of the review by Bhattacharya and Gupta [12], and Allton [14].)

Recent attempts to extract $m_{s}$ using QCD sum rules are of four types. Two employ flavorbreaking differences of vector and/or axial vector current correlators, either the isovectorhypercharge difference [3, [] or the isovector-strange difference [9 [1]. The others involve analyses of the strange scalar [2, [4] and strange pseudoscalar [2,8] channels.

For the vector current isovector-hypercharge difference, isospin-breaking corrections are crucial due to the high degree of cancellation on the hadronic side of the sum rule. These are, for natural reasons [7,24], larger than one would naively expect, and can only be obtained indirectly [7]. The high degree of cancellation also magnifies experimental and theoretical uncertainties, leading to significant errors on $m_{s}\left(m_{s}=107 \pm \sim 40 \mathrm{MeV}\right.$ [25], if one takes the isovector spectral function from hadronic $\tau$ decay data [26.27]).

The isovector-strange difference, which employs $\tau$ decay data for the isovector and strange spectral functions, is subject to complications resulting from (1) very poor convergence of the perturbative series for the longitudinal contributions on the OPE side [9:10], and (2) strong cancellation, which again significantly magnifies the effect of the (otherwise rather small) experimental errors. Combining errors in quadrature and translating to the scale 2 $\mathrm{GeV}$, one finds $m_{s}=163 \pm 50 \mathrm{MeV}$ [9], $143 \pm 42 \mathrm{MeV}$ [10] and $157 \pm 37 \mathrm{MeV}$ [11].

The third approach involves the correlator $\left\langle 0\left|T\left(J_{A, s} J_{A, s}^{\dagger}\right)\right| 0\right\rangle$ (where $J_{A, s} \equiv \partial_{\mu} \bar{s} \gamma_{5} \gamma^{\mu} u$ ) [2.88]. In this case, the $K$ pole contribution to $\rho(s)$ is known, but contributions from the continuum (K(1460), $K(1830)$ resonance region) are not. Both analyses of this type employ a sum-of-Breit-Wigner modulation of the near-threshold tree-level ChPT form to deal with this problem, together with some assumptions about the relative size of the two unknown resonance decay constants. As discussed in Refs. [0.28, this type of normalization procedure for a continuum spectral ansatz can involve potentially significant systematic errors. For 
example, in the scalar strange channel, it leads to a rather poor representation of the actual spectral function (compare the model versions of Refs. [2, (4] with that of Ref. [5]). In addition, because both analyses employ Borel transformed (SVZ) sum rules [29], they involve the conventional "continuum" (or local duality) approximation to $\rho(s)$ above $s=s_{0}$ (where the "continuum threshold", $s_{0}$, is a parameter of the analysis). Since, for Borel masses, $M$, in the stability windows of both analyses, $M^{2}<<s_{0}$ is far from being satisfied, there are potential systematic errors resulting from violations of local duality in the "continuum" region. (That contributions from the "continuum" region are not numerically negligible can also be inferred, e.g., from the figures of Ref. (8)). It is worth noting that violations of local duality are, in general, expected in kinematic regimes where typical resonance widths are comparable to or smaller than typical resonance separations. This has been quantified in the isovector vector channel, where the hadronic spectral function is known experimentally; violations of local duality in that channel, even at scales $\sim m_{\tau}^{2}$, are seen to be, in general, rather large [30]. Taking the results from Refs. [2, [4] which employ $\Lambda_{Q C D}=380 \mathrm{MeV}$ (which best corresponds to recent experimental values for $\alpha_{s}\left(m_{\tau}^{2}\right)$ [26.27]), one finds, for $m_{s}$, the values $\simeq 100 \mathrm{MeV}$ [2] (see Figure 5) and $115 \pm 19$ [8] (where the errors do not reflect the systematic uncertainties discussed above).

The last approach involves the correlator, relevant to the strange scalar channel,

$$
\begin{aligned}
\Pi\left(q^{2}\right) & =i \int d x e^{i q \cdot x}\left\langle 0\left|T\left(J_{s}(x) J_{s}^{\dagger}(0)\right)\right| 0\right\rangle \\
& =i\left(m_{s}-m_{u}\right)^{2} \int d x e^{i q \cdot x}\langle 0|T(\bar{s} u(x) \bar{u} s(0))| 0\rangle,
\end{aligned}
$$

which has been analyzed, in Refs. [2, [4], using the Borel transformed sum rule method. This channel is a favorable one for sum rule analyses because the hadronic spectral function, $\rho(s)$, turns out to be rather well-determinable (albeit indirectly) from experimental data. The reason is that since, experimentally, $K \pi$ scattering is known to be purely elastic up to $s=(1.58 \mathrm{GeV})^{2}$ [31,32], the phase of the timelike scalar $K \pi$ form factor, $d(s)$, is known up to this point. $d(s)$, moreover, satisfies an Omnes representation

$$
d(s)=d(0) p(s) \exp \left[\frac{s}{\pi} \int_{t h}^{\infty} d t \frac{\delta(t)}{t(t-s-i \epsilon)}\right],
$$

where $p(s)$ is a polynomial normalized to 1 at $s=0, \delta(s)$ is the phase of $d(s)$, and the normalization $d(0)$ is known from ChPT and $K_{e 3}$ [33]. In order to satisfy quark counting rules for the asymptotic behavior of $d(s), \delta$ must $\rightarrow(N+1) \pi$ as $s \rightarrow \infty$ if $p(s)$ is of degree $N$. In previous analyses $p(s)$ has been assumed to be 1 (or, equivalently, very slowly varying in the range of $s$ important to the analysis). The fact that, at the edge of the experimentally explored region, $s=(1.7 \mathrm{GeV})^{2}$, the $I=1 / 2 K \pi$ phase shift is very nearly $\pi$ [31,32,34], the asymptotic value required if $p(s) \equiv 1$, suggests, first, that this assumption is rather plausible and, second, that the treatment of the high energy behavior of the phase, $\delta(s)$, employed in Ref. [0], i.e. $\delta(s)=\pi$ for $s>(1.7 \mathrm{GeV})^{2}$, is sensible. We will also see below that, making these assumptions, one obtains extremely well-satisfied sum rules, providing strong post facto justification as well. With these assumptions, the $K \pi$ contribution to $\rho$ is completely determined, and multiparticle contributions begin only above $s \sim 2.5 \mathrm{GeV}^{2}$. The resulting $K \pi$ contribution to $\rho(s)$ is, moreover, much smaller in the $K_{0}^{*}(1950)$ than in 
the $K_{0}^{*}(1430)$ region (see Fig. 2 of Ref. [5]). Since the $K_{0}^{*}(1950) K \pi$ branching fraction is known to be $\sim 50 \%$ [35], the remaining multiparticle contributions to $\rho$, in the $K_{0}^{*}(1950)$ region, will also be small. This means that, to the extent that one may expect multiparticle contributions to $\rho$ to be resonance dominated, (1) such contributions will play only a small role in the full spectral function out to $s \sim 4 \mathrm{GeV}^{2}$, and (2) in this region, the magnitude of these contributions can be obtained by combining information from the Omnes-generated $K \pi$ component and the known experimental $K_{0}^{*}(1950) K \pi$ branching fraction. We do not quote results from the analyses of Refs. [2, [1] since the spectral ansatz employed there has been shown to be incompatible with the Omnes representation [5]. Results from Refs. [5],6] are $m_{s}=91 \rightarrow 116 \mathrm{MeV}$ [5] and $116 \pm 22 \mathrm{MeV}$ [6]. The main potential uncertainty in these analyses is that associated with violations of local duality in the "continuum" region, $s>s_{0}$. That continuum contributions are non-negligible may be seen from the lack of a stability plateau for $m_{s}$ in the analysis of Ref. [6]. Finite energy sum rules constructed using the extracted values of $m_{s}$ from Refs. [5, 6] and the Omnes-generated spectral ansatz of Ref. [5] (also used in Ref. [6]) as input have been shown to be moderately well satisfied [30]. The residual deficiencies could arise from either shortcomings in the spectral ansatz or approximations on the OPE side of the sum rules. We will investigate this question in what follows.

\section{THE STRANGE SCALAR CHANNEL ANALYSIS}

Unitarity and analyticity ensure that correlators like $\Pi\left(q^{2}\right)$, defined above, satisfy either appropriately subtracted conventional dispersion relations or finite energy sum rules (FESR's). Borel transformation of the former leads to the conventional [29] (SVZ) form of QCD sum rules. In what follows we choose to employ FESR's, which are based on Cauchy's theorem, rather than the Cauchy representation theorem, and work with the "Pac-man" contour, which traverses both sides of the physical cut, and is closed by a circle of radius $s_{0}$ in the complex-s plane. One then has

$$
\int_{s_{t h}}^{s_{0}} d s \rho(s) w(s)=\frac{-1}{2 \pi i} \oint_{|s|=s_{0}} d s w(s) \Pi(s)
$$

valid for any function $w(s)$ analytic in the region of the contour, where $\rho(s)$ is the spectral function and $s_{t h}$ the relevant physical threshold. To make this relation useful, one wishes to use the physical spectral function, whose extraction was described in the Introduction, on the LHS, and the OPE for the correlator on the RHS. Since the OPE is proportional to $\left(m_{s}-m_{u}\right)^{2}$, this provides a method for extracting $m_{s}-m_{u}$.

To make the results of this analysis reliable, $s_{0}$ should satisfy two criteria: (1) it should be large enough to make the OPE representation of the RHS accurate, and (2) it should be small enough that one still has experimental information on $\rho(s)$ for $s<s_{0}$ on the LHS. The latter criterion, in the present case, restricts us to the region up to and including the $K_{0}^{*}(1950)$ (i.e., $s_{0}<4 \mathrm{GeV}^{2}$ or so). Since, even at $s=4 \mathrm{GeV}^{2}$, resonance widths and separations are comparable, one expects local duality to be violated, creating potentially significant uncertainties on the OPE side. It has been argued, however, that even if local duality is not exactly satisfied for given timelike $s_{0}$, the OPE may, nonetheless, provide a good representation of the correlator on most of the circle $|s|=s_{0}$ (i.e., except possibly for 
a region of hadronic scale near the timelike real axis) [36]. The isovector vector channel, for which the spectral function has been extracted from hadronic $\tau$ decay data, provides explicit empirical confirmation of this argument. First, the conventional FESR treatment of these decays [37] is very well satisfied, presumably because it involves a weight function (determined by kinematics) with a double zero at $s=s_{0}$ which cuts out contributions from the potentially dangerous part of the contour. Second, one may demonstrate that using weights $s^{k}$ (with no such zero at $s=s_{0}$ ) leads to significant violation of local duality, even at scale $s_{0}=m_{\tau}^{2}$, whereas FESR's based on weights having either a single or double zero at $s=s_{0}$ are all extremely well satisfied, even at scales significantly below $s_{0}=m_{\tau}^{2}$ [30]. Using such FESR's one may, thus, hope to construct sum rules in which the use of the "continuum" ansatz for the high energy portion of $\rho(s)$ may be avoided.

In what follows, therefore, we study FESR's for the correlator, $\Pi\left(q^{2}\right)$, using weights of the form

$$
w_{A}(s)=\left(1-\frac{s}{s_{0}}\right)\left(A-\frac{s}{s_{0}}\right) .
$$

In Eq. (4), $A$ is a free parameter, to be chosen in such a way as to optimize the reliability of the analysis. Since (1) instanton effects are known exactly only in dilute gas singleinstanton-background approximation [38,39] and (2) the instanton density used to obtain these results is known to be altered by quark and gluon condensate effects [40], the magnitude of the resulting instanton contributions (as stressed by the authors of Refs. [38, 39]) can be considered only a rough guide. For this reason, we restrict our attention to weights which, after integration, yield instanton effects (including corrections to the coefficients of both the unit operator and quark condensate in the OPE, as given in Ref. [39]) which are small for all $s_{0}$ employed. While any $A>1$ turns out to satisfy this criterion, $A=2$ creates an optimal suppression and we will display results below for this value of $A$.

We now discuss the input on the hadronic side of the sum rules. The $K \pi$ and multiparticle portions of $\rho(s)$ are obtained, using the Omnes representation, experimental $K \pi$ phases, and the experimental $K \pi$ branching fractions of the $K_{0}^{*}(1430)$ and $K_{0}^{*}(1950)$, as described in the Introduction. We employ two slightly different implementations of this procedure, the difference in the resulting $m_{s}$ values serving to quantify the effect of uncertainties in our knowledge of the hadronic spectral function. In the first implementation, the fit to the experimental $K \pi$ phases obtained in Ref. [2] is used as input to the Omnes representation, and multiparticle contributions are allowed in both the $K_{0}^{*}(1430)$ and $K_{0}^{*}(1950)$ regions, constrained by the Particle Data Group's $K \pi$ branching fractions [35]. In the second implementation we employ the LASS fit [31.32] to the $K \pi$ phase shifts and inelasticities. For this fit, the form of the parametrization of phase shift data used is slightly different from that of Ref. [2]. In addition, since the fit to the $K \pi$ amplitude is still purely elastic in the $K_{0}^{*}(1430)$ region, in employing this implementation we allow multiparticle contributions to the spectral function only from the $K_{0}^{*}(1950)$ region. (Note that the background effective range parameters, $a, b$, and $K_{0}^{*}(1430)$ resonance parameters, $m, \Gamma$, were mis-recorded in Ref. [31, and should actually be $m=1.412 \mathrm{GeV}, \Gamma=.294 \mathrm{GeV}$ [32,41], and $a=2.19 \mathrm{GeV}^{-1}$, $b=3.74 \mathrm{GeV}^{-1}$ [32]. It has been checked that these provide a satisfactory representation of the amplitude and phase shift data of Ref. [31].)

On the OPE side, it is convenient to work with $d^{2} \Pi\left(Q^{2}\right) /\left(d Q^{2}\right)^{2} \equiv \Pi^{\prime \prime}\left(Q^{2}\right)$, which satisfies a homogenous RG equation, so all scale dependence can be absorbed into the running mass 
and coupling. To improve the convergence of the perturbative series, and significantly reduce residual scale dependence, we follow standard practice and evaluate the OPE side using the "contour improvement" prescription of Ref. [42], first summing logarithms through the renormalization scale choice $\mu^{2}=Q^{2}$, and then performing the integral around the circle $|s|=s_{0}$ numerically, using the exact solutions for the running mass and coupling which follow from the four-loop-truncated $\beta$ and $\gamma$ functions 43,44.

The expressions for the dimension $D=0,2,4,6$ contributions to $\Pi^{\prime \prime}\left(Q^{2}\right)$ are all known from previous work [2, 4, 5] and, for $\mu^{2}=Q^{2}$, are: (1) for $D=0$ [2, 四],

$$
\begin{array}{r}
{\left[\Pi^{\prime \prime}\left(Q^{2}\right)\right]_{D=0}=\frac{3\left(m_{s}-m_{u}\right)^{2}\left(Q^{2}\right)}{8 \pi^{2} Q^{2}}\left\{1+\frac{11 a\left(Q^{2}\right)}{3}+a\left(Q^{2}\right)^{2}\left(\frac{5071}{144}-\frac{35}{2} \zeta(3)\right)\right.} \\
\left.+a\left(Q^{2}\right)^{3}\left(\frac{1995097}{5184}-\frac{\pi^{4}}{36}-\frac{65869}{216} \zeta(3)+\frac{715}{12} \zeta(5)\right)\right\}
\end{array}
$$

where $a\left(Q^{2}\right)=\alpha_{s}\left(Q^{2}\right) / \pi$ and $\zeta(N)$ is the Riemann zeta function; (2) for $D=2$ [2, 4],

$$
\left[\Pi^{\prime \prime}\left(Q^{2}\right)\right]_{D=2}=-\frac{3\left(m_{s}-m_{u}\right)^{2}\left(Q^{2}\right) m_{s}^{2}\left(Q^{2}\right)}{4 \pi^{2} Q^{4}}\left\{1+\frac{28 a\left(Q^{2}\right)}{3}+a\left(Q^{2}\right)^{2}\left(\frac{8557}{72}-\frac{77}{3} \zeta(3)\right)\right\} ;
$$

(3) for $D=4$ [2,4],

$$
\begin{gathered}
{\left[\Pi^{\prime \prime}\left(Q^{2}\right)\right]_{D=4}=\frac{\left(m_{s}-m_{u}\right)^{2}\left(Q^{2}\right)}{Q^{6}}\left\{2\left\langle m_{s} \bar{u} u\right\rangle\left(1+\frac{23}{3} a\left(Q^{2}\right)\right)-\frac{1}{9} I_{G}\left(1+\frac{121}{18} a\left(Q^{2}\right)\right)\right.} \\
\left.+I_{s}\left(1+\frac{64}{9} a\left(Q^{2}\right)\right)-\frac{3}{7 \pi^{2}} m_{s}^{4}\left(Q^{2}\right)\left(\frac{1}{a\left(q^{2}\right)}+\frac{155}{24}\right)\right\},
\end{gathered}
$$

where $I_{G}$ and $I_{s}$ are the RG-invariant $D=4$ condensate combinations of Ref. 4 ; and (4) for $D=6$ [2],

$$
\left[\Pi^{\prime \prime}\left(Q^{2}\right)\right]_{D=6}=\frac{\left(m_{s}-m_{u}\right)^{2}\left(Q^{2}\right)}{Q^{8}}\left\{3 m_{s}\langle g \bar{u} \sigma F u\rangle-\frac{32}{9} \pi^{2} \rho_{V S A} a\left(\langle\bar{s} s\rangle^{2}+\langle\bar{u} u\rangle^{2}+9\langle\bar{s} s\rangle\langle\bar{u} u\rangle\right)\right\}
$$

where $\rho_{V S A}$ is a parameter describing the deviation of the four-quark condensates from their vacuum saturation values. In the above equations, factors of $m_{u, d}$ have been dropped, except in the overall normalization $\left(m_{s}-m_{u}\right)^{2}$.

For the input required on the OPE side of the sum rule we employ the following: $\alpha_{s}\left(m_{\tau}^{2}\right)=.334 \pm .022$ [26] (needed as initial condition for the RG running of $\alpha_{s}$ ); for the $D=4$ condensates, $\left\langle\alpha_{s} G^{2}\right\rangle=0.07 \pm .01 \mathrm{GeV}^{4}$ [45], $\left(m_{u}+m_{d}\right)\langle\bar{u} u\rangle=-f_{\pi}^{2} m_{\pi}^{2}$, and $0.7<r_{c} \equiv\langle\bar{s} s\rangle /\langle\bar{u} u\rangle<1$ [2]; for the $D=6$ condensates, $\langle g \bar{q} \sigma F q\rangle=\left(0.8 \pm 0.2 \mathrm{GeV}^{2}\right)\langle\bar{q} q\rangle$ 22 and $\rho_{V S A}=5 \pm 5$ (i.e., allowing, to be conservative, up to an order of magnitude deviation from vacuum saturation). We evaluate $m_{s}-m_{u}$ by optimizing the match of the OPE and hadronic sides of the sum rules in question in the range $3 \mathrm{GeV}^{2} \leq s_{0} \leq 4 \mathrm{GeV}^{2}$, for which the dominant $D=0$ contour-improved series is well behaved. To convert the extracted values of $m_{s}-m_{u}$ to those for $m_{s}$, we take the central ChPT value for $m_{s} / m_{u}$ [1]. 
The errors on the extracted value of $m_{s}$ associated with those on the input OPE parameters, together with their sources, are as follows (where we list only those cases where the error is greater than $0.1 \mathrm{MeV}$ ): due to $\alpha_{s}\left(m_{\tau}^{2}\right), \pm 1.0 \mathrm{MeV}$; due to $\rho_{V S A}, \pm 0.7 \mathrm{MeV}$; due to $\left\langle\alpha_{s} G^{2}\right\rangle, \pm 0.2 \mathrm{MeV}$; due to $r_{c}, \pm 0.2 \mathrm{MeV}$. More significant, on the OPE side, is the error associated with the truncation of the $D=0$ contributions at four-loop order. If we assume continued geometric growth of the expansion coefficients, including this estimate for the next order term lowers $m_{s}$ by $2.8 \mathrm{MeV}$. In the final results below we have included this estimate of the five-loop order term in arriving at the quoted value, and assigned an error of twice the difference between the four-loop result and five-loop estimate. If added in quadrature, this source of error swamps all others on the OPE side.

The sources and errors on $m_{s}$ associated with uncertainties on the hadronic side of the sum rules (where greater than $0.1 \mathrm{MeV}$ ) are as follows: due to the error on the $K_{0}^{*}(1430)$ $K \pi$ branching fraction [35] (in the case of the first implementation described above, where multiparticle contributions are allowed in the $K_{0}^{*}(1430)$ region), $\pm 3.5 \mathrm{MeV}$; due to that on $m_{K_{0}^{*}(1430)}, \pm 0.6 \mathrm{MeV}$; due to that on $\Gamma_{K_{0}^{*}(1430)}, \pm 3.8 \mathrm{MeV}$; and due to that on $\Gamma_{K_{0}^{*}(1950)}$, $\pm 0.4 \mathrm{MeV}$. There is also a $0.4 \mathrm{MeV}$ difference between the extracted central values for the two different implementations of the hadronic spectral ansatz; this uncertainty is, however, swamped by that associated with the treatment of multiparticle contributions in the $K_{0}^{*}(1430)$ region in the case of the first implementation. We treat the latter as a systematic error common to both hadronic implementations, even though it is, strictly speaking, not consistent to include it in the case of the second implementation, based on the LASS fit, which is purely elastic in the $K_{0}^{*}(1430)$ region.

Taking the average of the central values associated with the two different hadronic implementations, and adding errors in quadrature, we then arrive at our final result,

$$
m_{s}\left(4 \mathrm{GeV}^{2}\right)=(115.1 \pm 5.7 \pm 5.2) \mathrm{MeV},
$$

where the first error is associated with the OPE side of the sum rule (and is completely dominated by our estimate of the error due to trunctation of the $D=0$ series) and the second with uncertainties on the hadronic side (where errors associated with the inelasticity in the $K_{0}^{*}(1430)$ region and with the uncertainty in the $K_{0}^{*}(1430)$ width are roughly equally important and, again, dominate all other sources). This result corresponds to

$$
m_{s}\left(1 \mathrm{GeV}^{2}\right)=(158.6 \pm 7.9 \pm 7.2) \mathrm{MeV} .
$$

In Figure 1 we display the match between the OPE and hadronic sides of the $A=2$ sum rule in the fit window, for the optimized value of $m_{s}$. The agreement is obviously excellent, increasing our confidence in both the assumptions about the form of the Omnes representation of $d(s)$, and the value of $m_{s}$ that results.

\section{SUMMARY}

We have determined $m_{s}$ from a FESR analysis in which the hadronic side of the sum rules employed is well determined by experimental data, modulo questions about the presence or absence of a polynomial factor in the Omnes representation of $d(s)$ and the high energy behavior of the phase $\delta(s)$. The excellent agreement between OPE and hadronic sides shown 
in the Figure argues strongly, albeit post facto, for the reliability of the input assumptions $p(s)=1$ and $\delta(s)=\pi$ for $s>(1.7 \mathrm{GeV})^{2}$. We have attempted to make conservative estimates of the errors associated with uncertainties on the hadronic and OPE sides of the sum rules and conclude that $m_{s}\left(4 \mathrm{GeV}^{2}\right)=115 \pm 8 \mathrm{MeV}$, compatible with results from recent quenched lattice calculations. It is worth noting that systematic uncertainties in the Borel transformed sum rule approach associated with the use of the "continuum ansatz" for the high energy portion of the hadronic spectral function are potentially significant in size on the scale of the errors quoted above. In Ref. [4], for example, a variation of $\pm 9 \mathrm{MeV}$ in $m_{s}\left(1 \mathrm{GeV}^{2}\right)$ results from variations of $\pm 0.5 \mathrm{GeV}^{2}$ in the continuum threshold parameter, $s_{0}$. Such uncertainties are, of course, absent in the FESR approach, and help to keep the final errors small. 


\section{FIGURES}

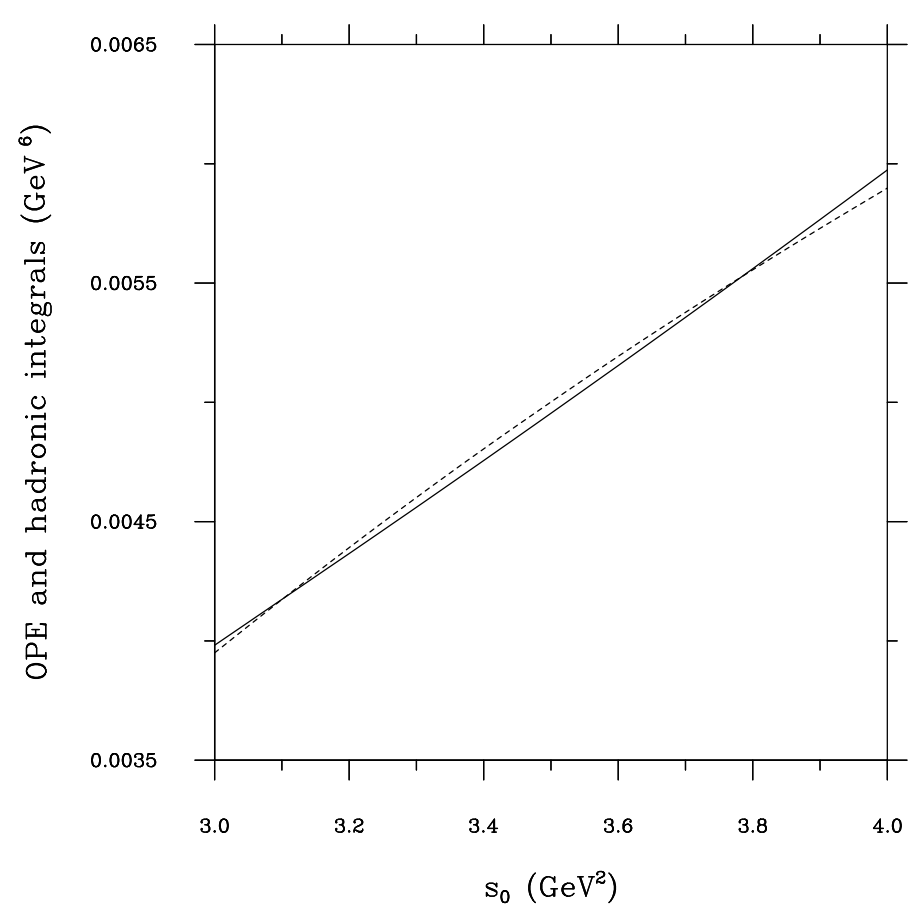

FIG. 1. The OPE $\left(I_{O P E}\right)$ and hadronic $\left(I_{h a d}\right)$ sides of the $A=2$ FESR defined in the text. The solid line gives the OPE side, the dashed line the hadronic side.

\section{ACKNOWLEDGMENTS}

The author would like to thank Rajan Gupta, Tanmoy Bhattacharya and Michael Pennington for numerous useful discussions; Michael Pennington, in addition, for pointing out the existence of the transcription errors in the $K \pi$ parameters of Ref. [31]; and W. Dunwoodie for confirming the revised values of these parameters. The ongoing support of the Natural Sciences and Engineering Research Council of Canada, and the hospitality and support of the Special Research Centre for the Subatomic Structure of Matter at the University of Adelaide, where much of this work was completed, are also gratefully acknowledged. 


\section{REFERENCES}

[1] H. Leutwyler, Phys. Lett. B374 (1996) 163; Phys. Lett. B378 (1996) 313 and hepph/9609467

[2] M. Jamin and M. Münz, Z. Phys. C66 (1995) 633

[3] S. Narison, Phys. Lett. B358 (1995) 113

[4] K.G. Chetyrkin, D. Pirjol and K. Schilcher, Phys. Lett. B404 (1997) 337

[5] P. Colangelo, F. De Fazio, G. Nardulli and N. Paver, Phys. Lett. B408 (1997) 340

[6] M. Jamin, Nucl. Phys. Proc. Suppl. 64 (1998) 250

[7] K. Maltman, Phys. Lett. B428 (1998) 179

[8] C. Dominguez, L. Pivovarov and K. Schilcher, Phys. Lett. B425 (1998) 193

[9] K. Maltman, Phys. Rev. D58 (1998) 093015

[10] A. Pich and J. Prades, J. High Energy Phys. 06 (1998) 013

[11] S. Chen, M. Davier and A. Höcker, to appear in Proceedings of the 5th International Workshop on Tau-Lepton Physics: Tau'98, Santander, Spain, 14-17 Sept. 1998

[12] R. Gupta and T. Bhattacharya, Phys. Rev. D55 (1997) 7203; Nucl. Phys. B (Proc. Suppl.) 63 (1998) 45

[13] C. Allton et al., Nucl. Phys. B489 (1997) 427

[14] C. Allton, Nucl. Phys. B (Proc. Suppl.) 64 (1998) 242

[15] B. Gough et al., Phys. Rev. Lett. 79 (1997) 1622

[16] N. Eicker et al. (The SESAM Collaboration), Phys. Lett. B407 (1997) 290; Phys. Rev. D59 (1999) 014509

[17] S. Aoki et al. (The CP-PACS Collaboration), hep-lat/9809120; hep-lat/9809146; heplat/9810043

[18] D. Becirevic et al., hep-lat/9809187

[19] M. Gockeler et al. (The QCDSF Collaboration), hep-lat/9810006, and as quoted in Ref. [20] below

[20] R.D. Kenway, hep-lat/9810054

[21] V. Gimenez et al., Nucl. Phys. B540 (1999) 472; hep-lat/9810532

[22] S. Aoki et al. (The JLQCD Collaboration), hep-lat/9901019

[23] T. Blum, A. Soni and M. Wingate, hep-lat/9902016

[24] K. Maltman, Phys. Rev. D58 (1998) 014008

[25] K. Maltman and C.E. Wolfe, hep-ph/9810441, to appear Phys. Rev. D

[26] R. Barate et al. (The ALEPH Collaboration), Z. Phys. C76 (1997) 379; Eur. Phys. J. C4 (1998) 409

[27] G. Abbiendi et al. (The OPAL Collaboration), Phys. Lett. B447 (1999) 134; Eur. Phys. J. C7 (1999) 571

[28] T. Bhattacharya, R. Gupta and K. Maltman, Phys. Rev. D57 (1998) 5455

[29] M.A. Shifman, A.I. Vainshtein and V.I. Zakharov, Nucl. Phys. B147 (1979) 385, 448

[30] K. Maltman, Phys. Lett. B440 (1998) 367

[31] D. Aston et al., Nucl. Phys. B296 (1988) 493

[32] W. Dunwoodie, private communication

[33] J. Gasser and H. Leutwyler, Nucl. Phys. B250 (1985) 517

[34] P. Estabrooks et al., Nucl. Phys. B133 (1978) 490

[35] Review of Particle Properties, Eur. Phys. J. C3 (1998) 1

[36] E. Poggio, H. Quinn and S. Weinberg, Phys. Rev. D13 (1976) 1958 
[37] E. Braaten, S. Narison and A. Pich, Nucl. Phys. B373 (1992) 581; F. Le Diberder and A. Pich, Phys. Lett. B286 (1992) 147; K.G. Chetyrkin and A. Kwiatkowski, Z. Phys. C59 (1993) 525; S. Narison and A. Pich, Phys. Lett. B304 (1993) 359; A. Pich, Nucl. Phys. Proc. Suppl. 39BC (1995) 326; S. Narison, Nucl. Phys. Proc. Suppl. 40B (1997) 47

[38] I.I. Balitsky, M. Beneke and V.M. Braun, Phys. Lett. B318 (1993) 371

[39] P. Nason and M. Palassini, Nucl. Phys. B444 (1995) 310

[40] M.A. Shifman, A.I. Vainshtein and V.I. Zakharov, Nucl. Phys. B163 (1980) 46; Nucl. Phys. B165 (1980) 45

[41] S. Godfrey and J. Napolitano, hep-ph/9811410

[42] F. Le Diberder and A. Pich, Phys. Lett. B289 (1992) 165

[43] T. van Ritbergen, J.A.M. Vermaseren and S.A. Larin, Phys. Lett. B400 (1997) 379

[44] K.G. Chetyrkin, Phys. Lett. B404 161 (1997) 161; T. Van Ritbergen, J.A.M. Vermaseren and S.A. Larin, Phys. Lett. B405 (1997) 327

[45] S. Narison, "International Europhysics Conference on High Energy Physics: Proceedings", Ed. D. Lellouch, G. Mikenber, and E. Rabniovici, Springer-Verlag, 199, pg. 388. 